\title{
A!
}

This is an electronic reprint of the original article.

This reprint may differ from the original in pagination and typographic detail.

Ross, G.; Vuorinen, V.; Petzold, M.; Paulasto-Kröckel, M.; Brand, S.

Gigahertz scanning acoustic microscopy analysis of voids in Cu-Sn micro-connects

Published in:

Applied Physics Letters

DOI:

$10.1063 / 1.4975305$

Published: 01/01/2017

Document Version

Publisher's PDF, also known as Version of record

Please cite the original version:

Ross, G., Vuorinen, V., Petzold, M., Paulasto-Kröckel, M., \& Brand, S. (2017). Gigahertz scanning acoustic microscopy analysis of voids in Cu-Sn micro-connects. Applied Physics Letters, 110(5), [054102].

https://doi.org/10.1063/1.4975305

This material is protected by copyright and other intellectual property rights, and duplication or sale of all or part of any of the repository collections is not permitted, except that material may be duplicated by you for your research use or educational purposes in electronic or print form. You must obtain permission for any other use. Electronic or print copies may not be offered, whether for sale or otherwise to anyone who is not an authorised user. 


\section{Gigahertz scanning acoustic microscopy analysis of voids in Cu-Sn micro-connects}

G. Ross, V. Vuorinen, M. Petzold, M. Paulasto-Kröckel, and S. Brand

Citation: Appl. Phys. Lett. 110, 054102 (2017); doi: 10.1063/1.4975305

View online: https://doi.org/10.1063/1.4975305

View Table of Contents: http://aip.scitation.org/toc/apl/110/5

Published by the American Institute of Physics

\section{Articles you may be interested in}

Quasi-free-standing bilayer graphene nanoribbons probed by electronic transport

Applied Physics Letters 110, 051601 (2017); 10.1063/1.4975205

Photoacoustic eigen-spectrum from light-absorbing microspheres and its application in noncontact elasticity evaluation

Applied Physics Letters 110, 054101 (2017); 10.1063/1.4975373

Suppressing excitation effects in microwave induced thermoacoustic tomography by multi-view Hilbert transformation

Applied Physics Letters 110, 053701 (2017); 10.1063/1.4975204

Crystallinity dependent thermal degradation in organic solar cell

Applied Physics Letters 110, 053301 (2017); 10.1063/1.4975140

Strong coupling between Tamm plasmon polariton and two dimensional semiconductor excitons Applied Physics Letters 110, 051101 (2017); 10.1063/1.4974901

Water-driven actuation of Ornithoctonus huwena spider silk fibers

Applied Physics Letters 110, 053103 (2017); 10.1063/1.4974350

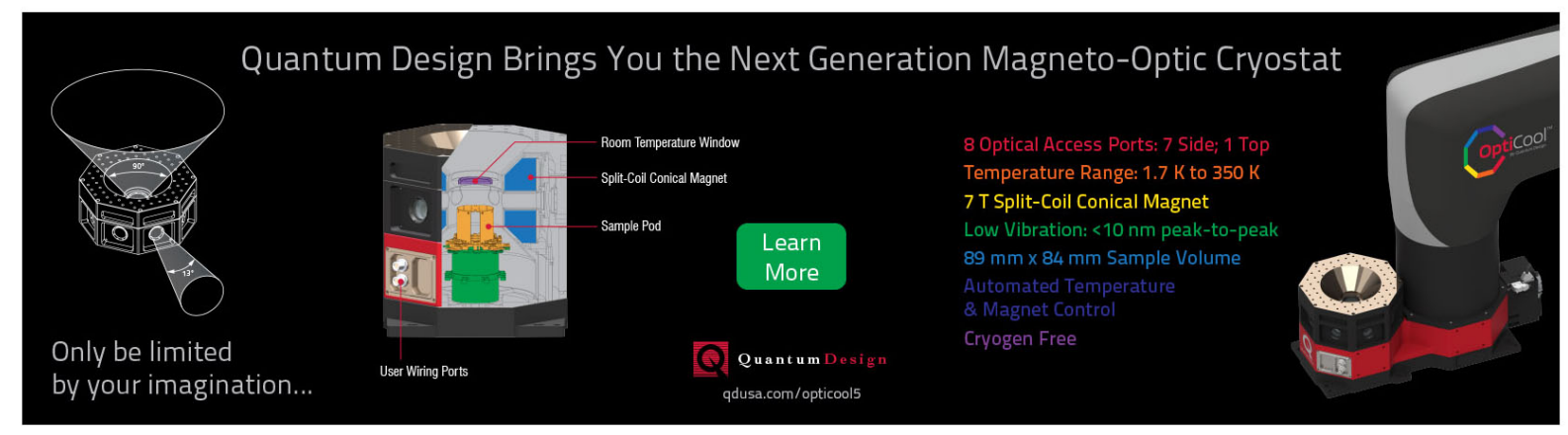




\title{
Gigahertz scanning acoustic microscopy analysis of voids in Cu-Sn micro-connects
}

\author{
G. Ross, ${ }^{1, a)}$ V. Vuorinen, ${ }^{1}$ M. Petzold,${ }^{2}$ M. Paulasto-Kröckel, ${ }^{1}$ and S. Brand ${ }^{2}$ \\ ${ }^{1}$ Department of Electrical Engineering and Automation, Aalto University, P.O. Box 13500, FIN-00076 Aalto, \\ Finland \\ ${ }^{2}$ Fraunhofer Institute for Microstructure of Materials and Systems IMWS, Halle (Saale), Germany
}

(Received 9 November 2016; accepted 19 January 2017; published online 2 February 2017)

\begin{abstract}
Gigahertz scanning acoustic microscopy (GHz-SAM) is applied to the characterization of bulk voids in the $\mathrm{Cu}-\mathrm{Sn}$ material system, often used in micro-connects. An increased demand for the development of miniaturized interconnect technologies, such as micro-connects, means that fast characterization methods are required for the assessment and detection of reliability impacting defects. This study attempts to formulate an analytical technique aimed at detecting microstructural defects in $\mathrm{Cu}-\mathrm{Sn}$ micro-connects, such as micro-bumps for 1st level interconnects and solid-liquid interdiffusion bonds for nano- and microelectromechanical systems. To study the potential of the analytical method, a specific electroplating chemistry was used that increases the probability of defect formation in the electroplated $\mathrm{Cu}$ film. The chemistry is known under certain electroplating overpotentials to promote hydrogen bubble induced voids within the $\mathrm{Cu}$. The samples containing voids were inspected by GHz-SAM with a highly focused acoustic lens operating at $1.12 \mathrm{GHz}$. To validate the results, GHz-SAM micrographs were compared with focused ion beam prepared cross-sections of the selected samples. Advances in acoustic transducer technology operating in the $\mathrm{GHz}$ frequency band allow for micron sized defect examination of materials with enhanced lateral resolution and sub-surface sensitivity. Published by AIP Publishing.
\end{abstract}

[http://dx.doi.org/10.1063/1.4975305]

Material defects in micro-connects, such as microbumps and solid-liquid interdiffusion (SLID) bonds for nano- and microelectromechanical systems (NEMS and MEMS), are a challenge to the implementation of high-end electronic integration and packaging technologies. ${ }^{1-3}$ There are two major factors influencing the effective implementation of these technologies. First, voids are known to reduce both the electrical and mechanical performance of microconnects, ${ }^{4,5}$ as voids can occupy a significant volume fraction of an interconnect. Second, a lack of understanding as to how voids evolve over the lifetime of an interconnect necessitates the development of reliable void detection and characterization methods for micro-connects.

Traditional detection and characterization of voids is commonly performed by employing cross-section imaging techniques, such as mechanical cross-sectioning or Focused Ion Beam (FIB) milling followed by high resolution imaging. These methods are often long and cumbersome, and they represent only one cross-sectional plane of an entire micro-connect, which renders them incompatible with backend screening processes during manufacturing. A fast and accurate method is required for detecting micron to sub-micron sized defects. Scanning Acoustic GigahertzMicroscopy (GHz-SAM) appears to be a promising technique for the detection of such microstructural defects. ${ }^{6-10}$ This work presents the application of GHz-SAM for the detection of void defects in electroplated $\mathrm{Cu}$ layers that required only minor sample preparation. Results show that

${ }^{\text {a) Electronic mail: glenn.ross@aalto.fi }}$ while performing a defocusing sweep of the acoustic lens, voids can be detected within a $\mathrm{Cu}-\mathrm{Sn}$ system.

Samples were prepared on a $100 \mathrm{~mm}$ diameter Si wafer. A $120 \mathrm{~nm}$ layer of $\mathrm{SiO}_{2}$ was deposited using PECVD followed by a layer stack of $100 \mathrm{~nm} \mathrm{Pt} / 100 \mathrm{~nm} \mathrm{Cr} / 100 \mathrm{~nm} \mathrm{Cu}$ deposited by magnetron sputtering. The $\mathrm{Cu}$ electroplating solution used in this work was a polyethylene glycol (PEG) based solution that consisted of $0.25 \mathrm{M} \mathrm{CuSO} \cdot 5 \mathrm{H}_{2} \mathrm{O}$ (Aldrich 209198), $1.8 \mathrm{M} \mathrm{H}_{2} \mathrm{SO}_{4}$ (Aldrich 40306), $1.92 \mathrm{mM} \mathrm{HCl}$ (Aldrich 40304), and $0.059 \mathrm{mM}$ of PEG or $\mathrm{H}\left(\mathrm{OCH}_{2} \mathrm{CH}_{2}\right)_{\mathrm{n}} \mathrm{OH}$ (Aldrich 202444). $\mathrm{A} \mathrm{Cu}$ target thickness of $3 \mu \mathrm{m}$ was desired using a DC current density of $10 \mathrm{~mA} / \mathrm{cm}^{2}$. It has been reported ${ }^{11,12}$ that the PEG additive, under certain electroplating overpotentials, has been associated with the adhesion of hydrogen bubbles on the cathode surface. Hydrogen bubbles inhibit the growth of $\mathrm{Cu}$ during the electroplating process ${ }^{13}$ and are the cause of the voids in this study. Following the $\mathrm{Cu}$ deposition, a $3 \mu \mathrm{m}$ layer of electroplated Sn was deposited. Once the sample structure was complete, the wafer was diced into $10 \mathrm{~mm} \times 10 \mathrm{~mm}$ squares. The samples were then annealed for $24 \mathrm{~h}$ at a temperature of $150{ }^{\circ} \mathrm{C}$ below the melting point of Sn to allow for solid state interdiffusion to occur and to form the $\mathrm{Cu}-\mathrm{Sn}$ intermetallic compounds (IMCs) of $\mathrm{Cu}_{3} \mathrm{Sn}$ and $\mathrm{Cu}_{6} \mathrm{Sn}_{5}$ with thicknesses in the order of $1 \mu \mathrm{m} .{ }^{14}$ To reduce the acoustic energy losses caused by a high sample thickness and to avoid diffuse scattering of the acoustic waves at the rough Sn layer, the samples were imaged through the Pt surface of the $\mathrm{Cu}$-Sn sample. ${ }^{15}$ Therefore, the sample films were detached from the Si substrates at the Pt surface by exfoliation. Exfoliation was performed by attaching (Loctite ${ }^{\circledR}$ power epoxy) the Sn surface to a brass plate, and the $\mathrm{Si} / \mathrm{SiO}_{2}$ substrate was exfoliated with 
sticky tape. An overview of the sample preparation steps is provided in Fig. 1.

For acoustic inspection, a GHz-SAM developed in close collaboration between Fraunhofer IMWS, Halle, Germany, and PVA TePla Analytical Systems was employed. The GHz-SAM was equipped with a $1 \mathrm{GHz}$ transducer with an opening angle of $100^{\circ}$ and a working distance between the acoustic lens and the sample surface of $80 \mu \mathrm{m}$. The acoustic wavelength in polycrystalline $\mathrm{Cu}$ using a sound velocity ${ }^{15}$ of $4760 \mathrm{~m} / \mathrm{s}$ at a frequency of $1 \mathrm{GHz}$ is approximately $4.76 \mu \mathrm{m}$. This setup provided a numerical acoustic aperture of 0.77 that allows for a lateral imaging resolution in $1 \mu \mathrm{m}$ range at the surface of the sample, spreading to $1-4 \mu \mathrm{m}$ underneath the surface, depending on the defocusing of the acoustic lens relative to the sample surface. However, this large numerical aperture also results in a restricted depth of field that leads to a limited imaging depth. A short focal length of the acoustic objectives operating in the GHz-band is required to limit the acoustic attenuation in the coupling fluid, ${ }^{16}$ which was deionized and degassed water at $21^{\circ} \mathrm{C}$. The application of a coupling fluid is required for coupling the acoustic energy from the probe to the sample, while allowing for mechanical scanning. It should be noted that the presence of a gas in the propagation path would lead to the total reflection of the acoustic wave at the solid/gas interface.

Data acquisition in the current study was performed in the $\mathrm{V}(\mathrm{z})$ mode, meaning that data frames (in $\mathrm{x}-\mathrm{y}$ ) were recorded repeatedly at decreasing distances (in z) between the acoustic lens and the sample, which allows for placing the acoustic focus sequentially on and below the sample surface and therefore allowing for the highest possible imaging resolution at the surface and sub-surface regions. The restricted depth of field and the corresponding limited penetration depth of the acoustic objective used here may be considered disadvantageous for the inspection of larger depths $(>10 \mu \mathrm{m})$, but they provide a high sensitivity at the surface and sub-surface regions down to approximately $6 \mu \mathrm{m}$, depending on the acoustically relevant material properties like elasticity, viscosity, mass-density, and structural composition. ${ }^{17}$ When defocusing, the probe is moved stepwise towards the surface of the sample. This process has two consequences: first, the focus of the acoustic field is placed underneath the sample surface, and second, the sample surface moves in the near field of the acoustic lens, which is where the wave fronts are increasingly curved leading to an angular incidence of the waves at the sample surface. As a consequence, several acoustic modes can be excited such as Rayleigh waves, transverse wave modes, or skimming surface compressional waves, which contribute to an increasingly complex acoustic field inside the sample which influences the image formation. Due to the strong curvature of the wave fronts at the sample surface (when placing the acoustic focus inside the sample), individual wave components arrive at a range of angles of up to $35^{\circ}$. As a consequence of this, in combination with the high refractive index $(\mathrm{n}=3.2$ for a water $-\mathrm{Cu}$ interface), the focus does not move equally into the solid as the lens approaches the sample surface. It should also be noted that the shape of the acoustic (i)

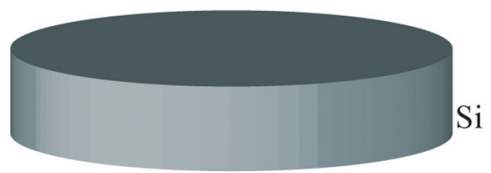

(iii)

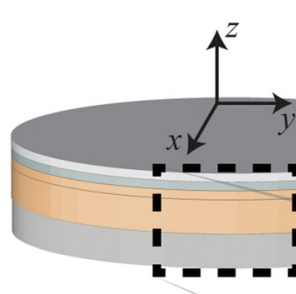

(v)

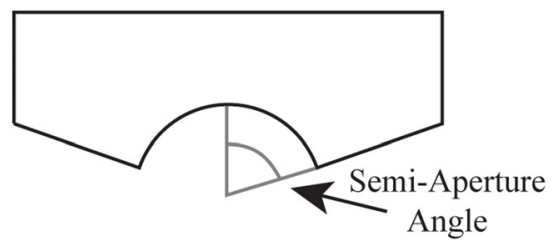

(ii)

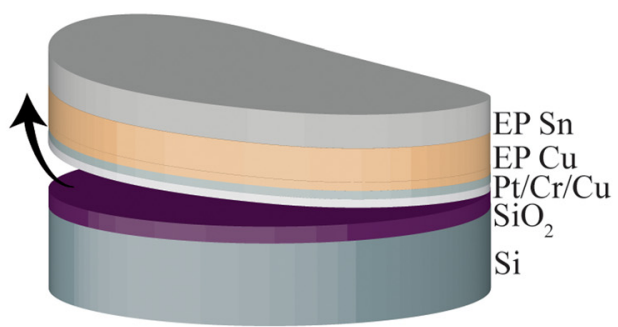

(iv)

Acoustic Lens

Coupling Fluid

$\mathrm{Cu} / \mathrm{Cr} / \mathrm{Pt}$

$\mathrm{EP} \mathrm{Cu}$

$\mathrm{Cu}_{3} \mathrm{Sn}$

$\mathrm{Cu}_{6} \mathrm{Sn}_{5}$

EP Sn
FIG. 1. Illustration of the sample preparation and the acoustic lens. (i) $\mathrm{Si}$ substrate; (ii) $100 \mathrm{~nm}$ of PECVD $\mathrm{SiO}_{2}$, magnetron sputtering of $100 \mathrm{~nm}$ of $\mathrm{Pt} /$ $\mathrm{Cr} / \mathrm{Cu}, 3 \mu \mathrm{m}$ of electroplated $\mathrm{Cu}, 3 \mu \mathrm{m}$ of electroplated $\mathrm{Sn}$, and removal of the $\mathrm{Si}$ substrate; (iii) final structure with the Cartesian coordinate system inset; (iv) final structure thermally annealed at $423 \mathrm{~K}\left(150^{\circ} \mathrm{C}\right)$ for $24 \mathrm{~h}$ to form the $\mathrm{Cu}-\mathrm{Sn}$ IMCs with the schematic of the acoustic lens for the sample inspection; and (v) overview of the aperture of the acoustic lens. 
beam varies during the defocusing sequence non-linearly. By definition, defocusing is performed in the negative $\mathrm{z}$ direction when the transducer is moved towards the sample. The reference $\mathrm{z}=0$ is defined as the surface of the sample being in focus of the acoustic lens. Z-values mentioned in the results correspond to the transducer displacement relative to the sample surface and should not be mistaken with the position of the acoustic focus inside the sample.

For analyzing and comparing the results obtained by acoustic GHz-microscopy, a simplified texture analysis software tool was developed and implemented in a custom software toolbox based on MATLAB (The Mathworks, Nattick, MA, USA). Upon manually selecting an upper and lower intensity-threshold, the texture in a defined region of interest (ROI) is binarized. Using MATLAB's image processing toolbox, connected objects are detected and their sizes are estimated. By performing a simple statistical analysis, the total number of objects of size greater than 8 pixels and their average size and size variation are calculated in $\mu \mathrm{m}^{2}$. For verification of the results obtained by acoustic $\mathrm{GHz}$ microscopy, a complementary study by FIB milling and scanning electron microscopy (SEM) imaging was performed using a NVISION FIB-SEM (Carl-ZeissMicroscopy, Oberkochen, Germany). First, an "L"-shaped marker was FIB-milled at a nonspecific location on the sample, and then a $200 \mu \mathrm{m} \times 200 \mu \mathrm{m}$ region around this marker was inspected by acoustic GHz-microscopy to assess the void distribution in the marker proximity. Locations for two cross-sections to be prepared by FIB were defined and trenches were prepared and polished for SEM imaging. To identify the exact trench-position, acoustic GHz-micrographs of the sample containing the FIB-trenches were recorded and superimposed on the GHz-SAM micrographs recorded before the trenching. This allowed precise alignment of the features imaged by GHz-SAM with the objects revealed by SEM imaging.

Imaging with maximum lateral resolution at the surface and sub-surface was performed in a $\mathrm{V}(\mathrm{z})$ sequence, resulting in a stack of 52 data frames. Fig. 2 contains a selection of the acoustic GHz-micrographs at increasing defocus (separation between the acoustic lens and the sample surface). Starting at $\mathrm{z}=-1 \mu \mathrm{m}$ in the upper left frame of Fig. 2, features slightly beneath the surface are shown. At $\mathrm{z}=-8 \mu \mathrm{m}$ and $\mathrm{z}=-15 \mu \mathrm{m}$, a large number of bright spots are observed. At $\mathrm{z}=-28 \mu \mathrm{m}$ and $\mathrm{z}=-39 \mu \mathrm{m}$, a large number of dark spots are seen. Also at $\mathrm{z}=-39 \mu \mathrm{m}$, the background shows an inhomogeneous cloud-pattern-distribution of bright and dark. Again, the defocus-values provided here correspond to the relative displacement of the acoustic lens with respect to the transducer's focus being at the sample surface. These values should not be mistaken with the position of the acoustic focus inside the sample. Fig. 3 contains the results of the feature detection and analysis. Using the acoustic $\mathrm{GHz}$ frame recorded at $\mathrm{z}=-15 \mu \mathrm{m}$, the bright spots are detected and their sizes of appearance are estimated based on the scanning parameters of the acoustic GHz-microscope. Objects detected by the custom analysis software are marked red. The total number of objects detected, their mean size of appearance, and the size variation (standard deviation) are provided in the label in Fig. 3. For comparison of features
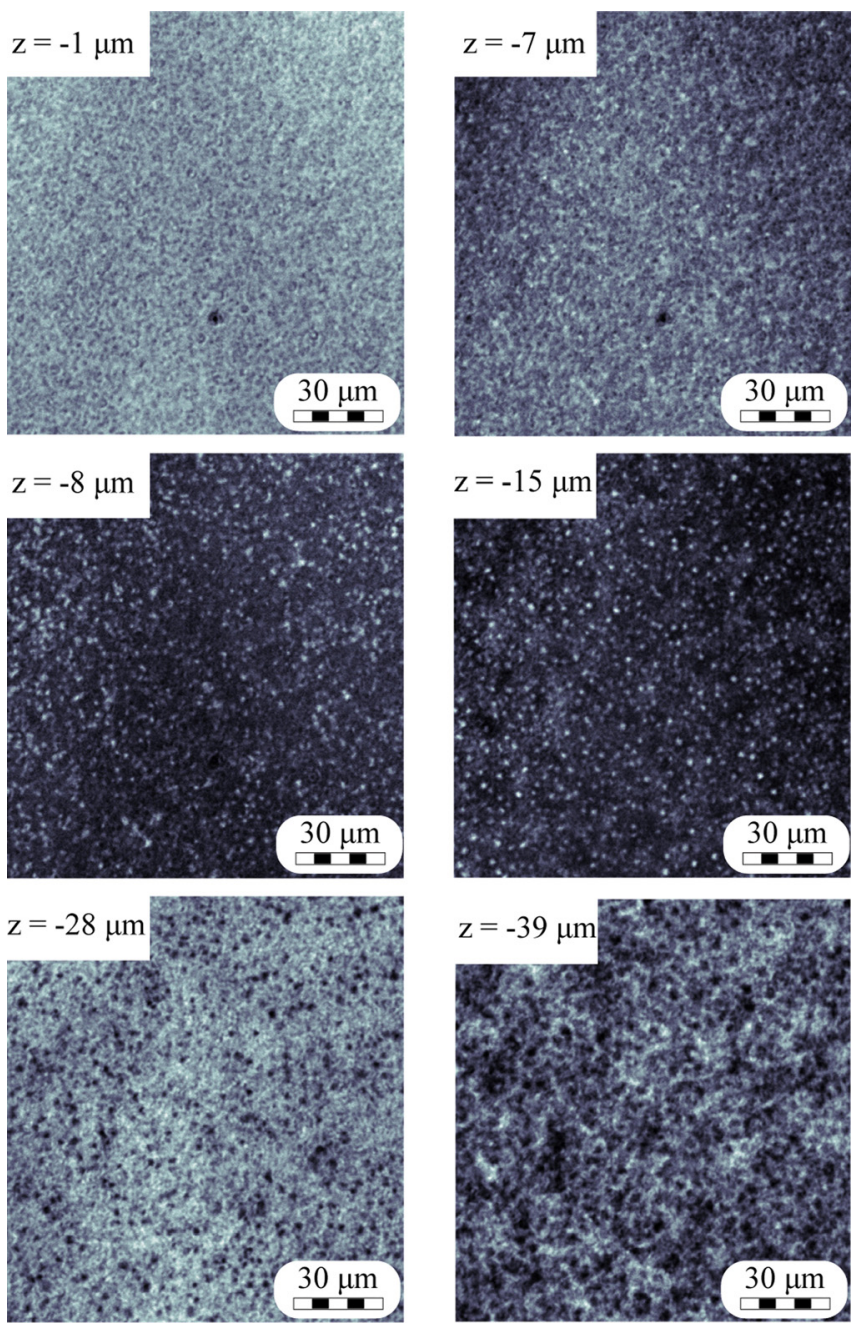

FIG. 2. Acoustic GHz-micrographs at increasing defocus (z values indicated correspond to the spacing between the acoustic lens and the sample, with $\mathrm{z}=0$ the sample surface being in focus). Contrast inversion between bright and dark features can be observed, likely indicating a shadowing effect.

between different samples and processing parameters, the results need normalization to the analyzed area within the ROI. In Fig. 3, an average of 43 objects per $100 \mu \mathrm{m}^{2}$ were detected. The number of detected objects with respect to their sizes of appearance, estimated by the analysis software, is presented in the histogram in Fig. 3(iii). A total of 558 objects have been identified; the majority $(\approx 230)$ of the objects have a size of appearance in the bin of the smallest size in the histogram. For confirmation and validation of the results, SEM micrographs were recorded from FIB-prepared cross sections and are shown in Fig. 4 together with the two corresponding acoustic GHz-micrographs. The four orange arrows in the $\mathrm{GHz}$-micrographs point to the location where the FIB cross-sections were prepared. The white and black arrows indicate the location of the voids, both in the $\mathrm{GHz}$ and SEM micrographs. It can be seen that the voids appear both bright and dark in the acoustic images, depending on the amount of defocus of the acoustic lens. The SEM images in the bottom row of Fig. 4 were recorded from the direction indicated by the bold yellow arrows in the acoustic GHzmicrographs, with the FIB-prepared trenches normal to the arrows (horizontal in the images within the orange marked 
(i)

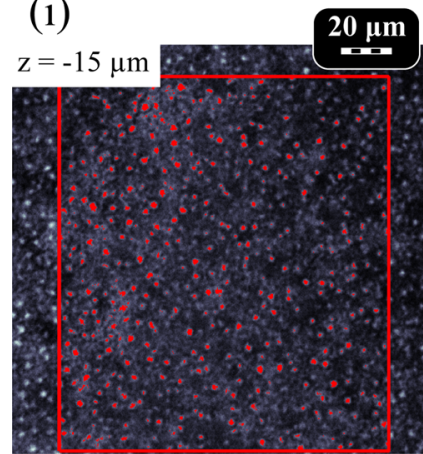

\# features detected per $100 \mu \mathrm{m}^{2}: 43$

Average size of appearance $\quad: 1.4 \pm 1.3 \mu \mathrm{m}^{2}$

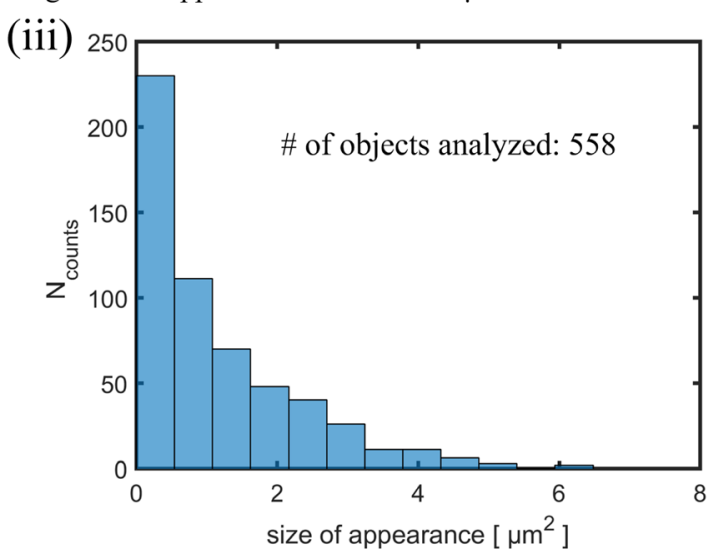

FIG. 3. Acoustic GHz-micrograph of a $\mathrm{Cu}-\mathrm{Sn}$ system, (i) at $\mathrm{z}=-15 \mu \mathrm{m}$ defocus, bright features can be observed which correspond to voids and (ii) $\mathrm{z}=-28 \mu \mathrm{m}$ defocus where dark features can be observed, which correspond to voids. Feature detection and analysis was performed on defocusing value $\mathrm{z}=-15 \mu \mathrm{m}$. Feature detection results overlaid (in red) in (i) report an average size of appearance of $1.4 \pm 1.3 \mu \mathrm{m}^{2}$. The feature detection was overlaid on the $\mathrm{z}=-28 \mu \mathrm{m}$ measurement illustrating not all objects appearing in $\mathrm{z}=-15 \mu \mathrm{m}$ occur dark in the $\mathrm{z}=-28 \mu \mathrm{m}$. The histogram seen in subfigure (iii) is from the object sizes (acoustic appearance) detected and analyzed by the feature detection algorithm.

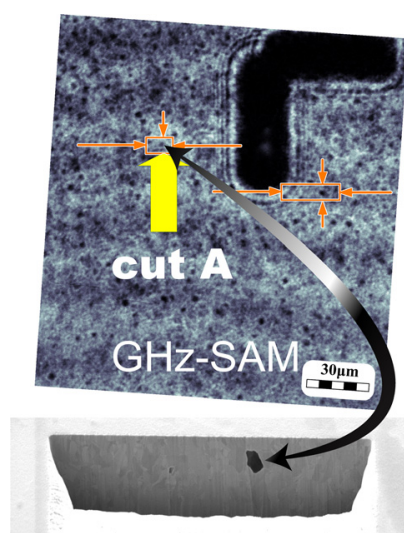

III

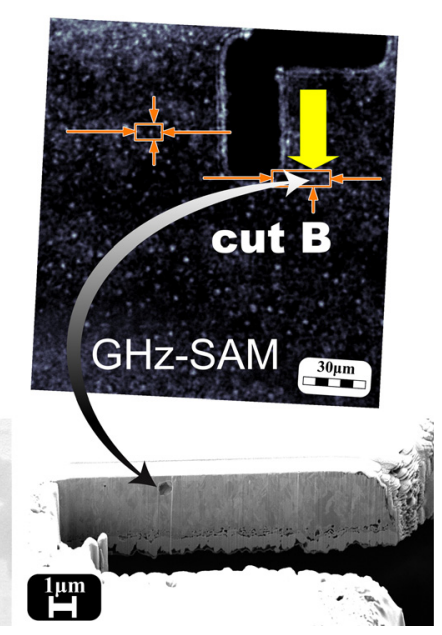

FIG. 4. Result validation by SEM images of FIB-prepared cross sections in direct comparison to the location in the GHz-micrographs. Top: GHz-micrographs with indication of the FIB prepared trenches (orange horizontal arrows). The yellow arrows indicate the direction of SEM imaging. Bottom: SEM micrographs of the cross sections. Voids in the $\mathrm{Cu}-\mathrm{Sn}$ system can clearly be seen. The voids of interest seen in the GHz-SAM and in the corresponding SEM micrographs are indicated with the white and black arrows. rectangle). Both SEM images clearly show a void located in the electroplated $\mathrm{Cu}$ that corresponds to the bright/dark spots in the GHz-micrographs.

By using GHz-SAM, the voiding behavior of $\mathrm{Cu}-\mathrm{Sn}$ micro-connects can be better evaluated and investigated. Fig. 3 shows a clear uniform distribution of voids within the imaging region, indicating that voiding occurs uniformly throughout the sample and does not form areas of void clustering. In addition to the distribution of the voids, their sizes can be estimated (within the resolution limits). Post processing of the acoustic data in Fig. 3 indicates an average size of appearance of the detected objects to be $1.4 \pm 1.3 \mu \mathrm{m}^{2}$. When acoustically imaging highly reflecting objects like gas or fluid filled inclusions in a solid matrix objects will appear with the size of the acoustic beam at that specific depth inside the solid when the objects are smaller than the resolution limits of the transducer. This is explained by the scanning imaging technique, which can be considered as a two-dimensional convolution of the acoustic beam with the highly reflective structure which allows for the detection of objects below the resolution limit. As seen from the histogram in Fig. 3(iii), the largest number of objects occur in the bin with the smallest object-size and thus seems to have dimensions below the resolution limit of the transducer. When the voids are larger and their dimensions exceed the resolution limit, their approximate size can be estimated. Assuming a stochastic distribution of the void sizes, a rather smoothly shaped distribution function is expected, as can be seen in the histogram in Fig. 3(iii) for size values larger than $0.5 \mu \mathrm{m}^{2}$. The conclusion to be drawn from these observations is that all voids with dimensions below the resolution limit but with properties above the detection limit are located in the bin with the smallest size values. When void dimensions increase and exceed the resolution limitations, their actual $\mathrm{x}$ y cross-sectional area can be estimated. Assuming a specific distribution function of void sizes, the sizes of the smaller voids may be extrapolated from the resolved void sizes at the right in the histogram (bins $>0.5 \mu \mathrm{m}^{2}$ ).

In conclusion, GHz-SAM has been presented in this work as a method for identifying and characterizing voids within electroplated $\mathrm{Cu}$-Sn films. Voids were seen in the GHz-SAM micrographs, both as a function in the $\mathrm{x}-\mathrm{y}$ plane and of the defocusing axis $z$. Although the estimated sizes are affected by the physical dimensions of the acoustic beam, especially for voids with sizes below the resolution limit of the acoustic GHz-transducer, the size distribution for objects larger than the imaging resolution can be estimated and may allow extrapolation of the lower size values by using a specific distribution function. Voids in electroplated interconnects represent a number of significant challenges for the electronic integration industry. The first is the sporadic nature of the phenomena. Second, the true impact of these voids is not entirely understood with conflicting reports on their impact on the electrical and mechanical integrity of small volume interconnects. Third, the problem is to find suitable characterization methods capable of non-destructively assessing voids in a volume of an electrical interconnect, which can then be further inspected using destructive analysis techniques for analyzing the root causes of their formation. 
The authors would like to thank EU Eniac, Finnish Funding Agency for Technology and Innovation, Okmetic, and Murata Electronics Oy for their financial support for the Lab4MEMS II (Grant No. 2013-2-621176) Project. Parts of this work have been conducted within the European Union's Horizon 2020 research and innovation program and were funded in the METRO4-3D Project under the Grant Agreement No. 688225.

${ }^{1}$ K. N. Tu, H. Y. Hsiao, and C. Chen, Microelectron. Reliab. 53, 2 (2013). ${ }^{2}$ J. H. Lau, J. Electron. Packag. 136, 40801 (2014).

${ }^{3}$ C. T. Ko and K. N. Chen, Microelectron. Reliab. 53, 7 (2013).

${ }^{4}$ H. H. Hsu, S. Y. Huang, T. C. Chang, and A. T. Wu, Appl. Phys. Lett. 99, 251913 (2011).

${ }^{5}$ K. Zeng, R. Stierman, T. C. Chiu, D. Edwards, K. Ano, and K. N. Tu, J. Appl. Phys. 97, 024508 (2005).

${ }^{6}$ S. Brand, T. Appenroth, F. Naumann, W. Steller, M. J. Wolf, P. Czurratis, F. Altmann, and M. Petzold, in Proceedings of the Electronic Components and Technology Conference (2015), p. 46.
${ }^{7}$ S. Brand, M. Simon-Najasek, M. Kögel, J. Jatzkowski, R. Portius, and F. Altmann, Microelectron. Reliab. 64, 341 (2016).

${ }^{8}$ A. Khaled, S. Brand, M. Kögel, T. Appenroth, and I. De Wolf, Microelectron. Reliab. 64, 336 (2016).

${ }^{9}$ S. Brand, A. Lapadatu, T. Djuric, P. Czurratis, J. Schischka, and M. Petzold, J. Micro/Nanolithogr., MEMS, MOEMS 13, 11207 (2014).

${ }^{10}$ A. Briggs, Advances in Acoustic Microscopy (Springer Science \& Business Media, 2013).

${ }^{11}$ B. Bozzini, C. Mele, L. D'Urzo, G. Giovannelli, and S. Natali, J. Appl. Electrochem. 36, 789 (2006).

${ }^{12}$ J. J. Kelly, J. Electrochem. Soc. 145, 3477 (1998).

${ }^{13}$ N. D. Nikolić, L. J. Pavlović, M. G. Pavlović, and K. I. Popov, Electrochim. Acta 52, 8096 (2007).

${ }^{14}$ G. Ross, V. Vuorinen, and M. Paulasto-Kröckel, J. Alloys Compd. 677, 127 (2016).

${ }^{15}$ A. Briggs, Acoustic Microscopy, in Monographs on the Physics and Chemistry of Materials, 47th ed. (Oxford University Press, Oxford, 1992).

${ }^{16}$ E. M. Strohm and M. C. Kolios, in 2011 IEEE International Ultrasonics Symposium (2011), pp. 2368-2371.

${ }^{17}$ A. Atalar, IEEE Trans. Sonics Ultrason. SU 32, 164 (1985). 